\title{
Erlotinib versus gefitinib for brain metastases in Asian patients with exon 19 EGFR-mutant lung adenocarcinoma: a retrospective, multicenter study
}

\author{
Ye Jiang ${ }^{1 \dagger}$, Jing Zhang ${ }^{2 \dagger}$, Juanjuan Huang ${ }^{3+}, \mathrm{Bo} \mathrm{Xu}^{4}$, Ning $\mathrm{Li}^{1}$, Lei $\mathrm{CaO}^{3}$ and Mingdong Zhao ${ }^{5^{*}}$
}

\begin{abstract}
Background: The purpose of this study was to compare clinical outcomes of Erlotinib versus Gefitinib in the treatment of Asian patients with exon 19 EGFR-mutant lung adenocarcinoma and newly diagnosed brain metastases.

Methods: Consecutive Asian patients with exon 19 EGFR-mutant lung adenocarcinoma and newly diagnosed brain metastases were identified and initially received peroral administration of $150 \mathrm{mg} / \mathrm{d}$ erlotinib or $250 \mathrm{mg} / \mathrm{d}$ gefitinib during 2009-2015. Overall survival (OS) was the primary endpoint. Progression-free survival (PFS) was the second endpoint.

Results: The cohort consisted of 227 Asian patients (erlotinib-treated cohort: $n=112$, mean age $=58.5$ years [SD: 20.13]; gefitinib-treated cohort: $n=115$, mean age $=58.4$ years [SD: 19.52]). In a multivariate analysis controlling for age, sex and time span of smoking history, significant difference was detected in the 36-month OS between erlotinib and gefitinib groups $(58.3 \%$ vs. $49.1 \%, p=0.012)$. There was also significant difference in the 36 -month PFS between erlotinib and gefitinib groups (64\% vs. 53\%, $p=0.013)$.

Conclusion: For Asian patients with exon 19 EGFR-mutant lung adenocarcinoma and brain metastases, erlotinib was associated with a significantly longer OS and a more prolonged PFS and compared with gefitinib.
\end{abstract}

Keywords: Erlotinib, Gefitinib, Lung adenocarcinoma, Overall survival

\section{Background}

Based on previous studies [1-4], gefitinib or erlotinib, epidermal growth factor receptor mutation - tyrosine kinase inhibitor (EGFR-TKI), has been a successful regimen managing advanced non-small cell lung cancer (NSCLC). Furthermore, the data from randomized controlled trials(RCTs) and other investigations have also indicated that EGFR-TKI has advantageous when used as an initial treatment for Asian patients with EGFR-mutant lung adenocarcinoma and brain metastases [5-7]. Yet overall survival (OS) and progression-free survival (PFS) remain controversial for Asian patients with exon

\footnotetext{
* Correspondence: zhaonissann@163.com

${ }^{\dagger}$ Ye Jiang, Jing Zhang and Juanjuan Huang contributed equally to this work. ${ }^{5}$ Department of Orthopaedics, Jinshan Hospital, Fudan University, Longhang Road No. 1508, Jinshan District, Shanghai City 201508, China

Full list of author information is available at the end of the article
}

19 EGFR-mutant lung adenocarcinoma and brain metastases [8-14].

We therefore conducted a retrospective review of Asian patients with exon 19 EGFR-mutant lung adenocarcinoma and brain metastases. To our knowledge, this is the first analysis that directly compares gefitinib against erlotinib as initial treatment for brain metastases following exon 19 EGFR-mutant lung adenocarcinoma. We hypothesized that there would be differences in both OS and PFS between patients treated with gefitinib vs. erlotinib.

\section{Materials and methods \\ Study population and end points}

The clinical and molecular characteristics and outcome data for 335 Asian patients with exon 19 EGFR-mutant lung adenocarcinoma and newly diagnosed brain metastases retrieved from a registry database were identified at the 4 medical centres between January 2009 and January

(c) The Author(s). 2018 Open Access This article is distributed under the terms of the Creative Commons Attribution 4.0 International License (http://creativecommons.org/licenses/by/4.0/), which permits unrestricted use, distribution, and 
2015. Information regarding erlotinib or gefitinib delivery, disease status and survival was obtained from the medical record. Inclusion criteria: age range: $50 \sim 70$ years; patients harbouring exon 19 EGFR mutation; all patients with stage IV lung adenocarcinoma at initial diagnosis; patients initially receiving peroral administration of $150 \mathrm{mg} / \mathrm{d}$ erlotinib or $250 \mathrm{mg} / \mathrm{d}$ gefitinib; EGFR mutation testing performed in all patients by the molecular diagnostic core laboratory of the Department of Pathology. Exclusion criteria: patients with de novo EGFR-TKI resistance mutations; previous chemotherapy or radiotherapy; no pre-treatment imaging; discontinuation or interruption of erlotinib or gefitinib; death; refusal; organ failure; severe infectious diseases (e.g., systemic inflammatory response syndrome); mental illness; cognitive dysfunction; uncontrolled diabetes mellitus or hypertension. OS was the primary endpoint. PFS was the second endpoint.

\section{Definitions of the descriptive variables}

OS was defined as the period from treatment initiation to the date of death from any cause. PFS was defined as the period from treatment initiation to the date of disease progression. Lung adenocarcinoma staging was performed according to the 7th edition of the Lung Cancer Stage Classification System [15]. For EGFR mutation testing, tumour specimens from primary lung adenocarcinoma were obtained by either needle biopsy/aspiration prior to EGFR-TKI therapy. Imaging examination was carried out every 2 months to assess the drug-related patient's response. Lung adenocarcinoma response was assessed in accordance with the Response Evaluation Criteria in Solid Tumours (RECIST) by imaging procedure 1 month after treatment and then every 2 months thereafter or when clinically indicated. Responses to EGFR-TKI were conducted by independent radiological reviews. For OS analyses, patients who were still alive or not lost to follow-up at the primary analysis cut-off date were noted at the final follow-up. Living patients were censored at the date of last contact. DNA which was extracted from formalin-fixed, paraffin-embedded tumour tissue was tested with polymerase chain reaction-based assays, as described by Pan et al. [16].

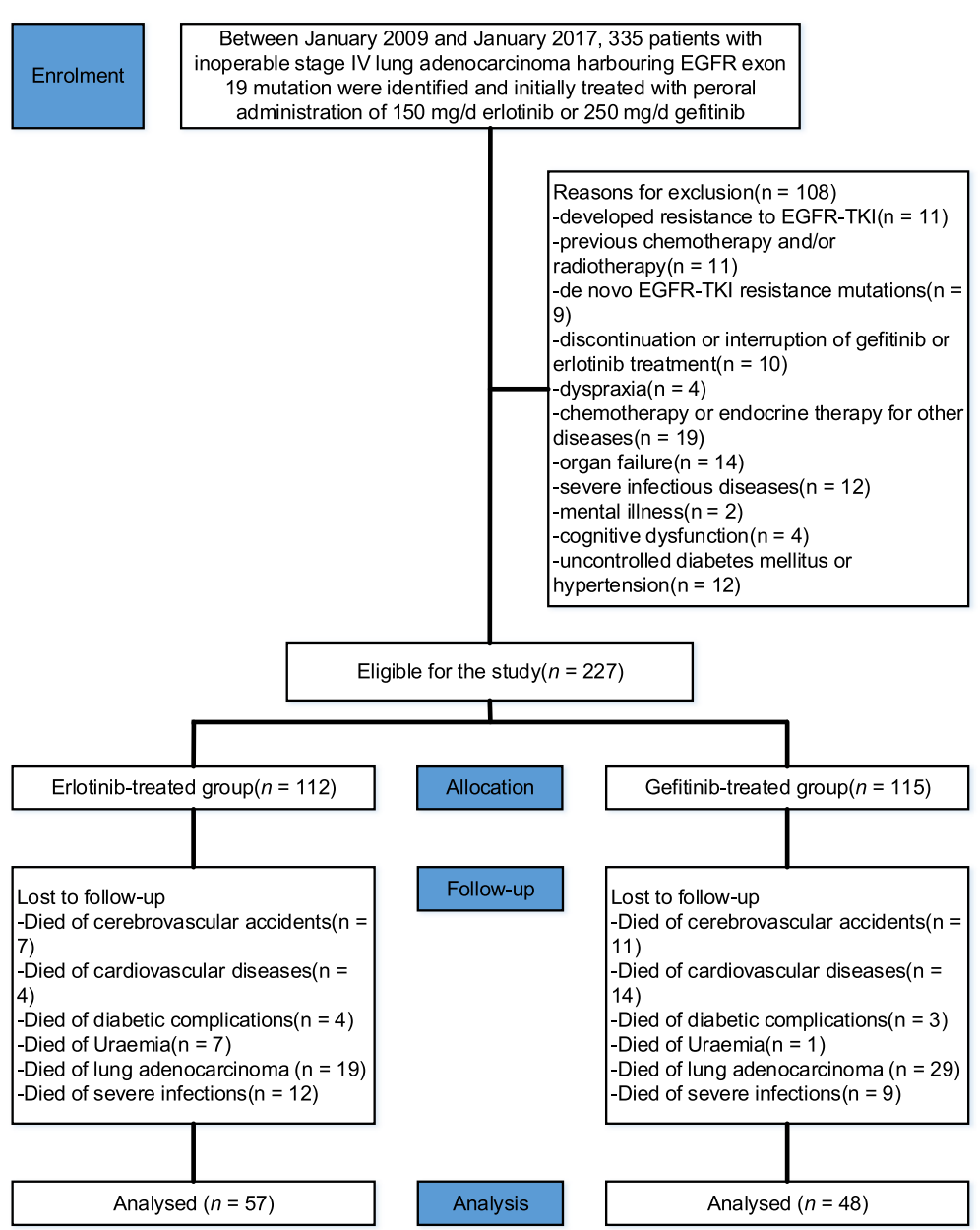

Fig. 1 Flow diagram demonstrating the methods to identify the study 


\section{Statistical analysis}

Categorical variables expressed as the count and percentage were analysed using $\chi^{2}$-test or the Mann-Whitney U-test. Continuous numeric variables expressed as the mean and SD were analysed with Student's t-test. Survival probabilities estimated using the Kaplan-Meier method were compared between groups by the log-rank test. Cox regression analyses were executed to adjust for age, sex and time span of smoking history. Statistical analyses were performed using SPSS (version 24.0; IBM, Inc., Chicago, IL, USA) software. A value of $p<$ 0.05 was considered statistically significant.

\section{Results}

\section{Patient characteristics}

In total, 227 Asian patients with exon 19 EGFR-mutant lung adenocarcinoma and brain metastases were included (erlotinib: $n=112$, mean age $=58.5$ years [SD: 20.13]; gefitinib: $n=115$, mean age $=58.4$ years [SD: 19.52]), as summarized in Fig. 1. The comparisons of the demographic characteristics are presented in Table 1. The median follow-up at the primary analysis cut-off date was 36 months (IQR: 14.5-39.6) for the erlotinib group and 36 months (IQR: 13.3-39.2) for the gefitinib group. The time to occurrence of the progression of brain tumours was significantly prolonged after erlotinib compared with gefitinib. No between-group significant differences were detected in regard to drug-related toxicity or intolerable adverse reactions.

\section{Survival analysis}

Deaths occurred in the erlotinib and gefitinib groups (44.6 and 58.3\%, respectively), as presented in Table 2. Twenty-seven cases had recurrences, 9 of which received the conversion from gefitinib to erlotinib, and no significant increase in brain metastases; 5 continued to receive gefitinib, and brain metastases further worsen until they nearly died; 2 terminated the treatment of gefitinib and eventually died; the therapy of 11 cases was unidentified. There were more than 3 metastases (the sites included the brain, bone, lung, liver, and lymph nodes) in 70 patients in the two groups ( 28 vs. 42 for erlotinib and gefitinib groups, respectively, $p=0.06$ ). All tumours detected were histopathologically parallel to lung adenocarcinoma with identical exon 19 EGFR mutation, excluding a second lung tumour as a possibility.

Median PFS and median OS of erlotinib-treated patients were 10.8 months (95\% CI: 4 to 16$)$ and 28.3 months (95\% CI: 3 to NA), respectively. Median PFS and median OS of gefitinib-treated patients were 8.4 months (95\% CI: 4 to 13 ) and 25.0 months (95\% CI: 5 to NA), respectively, as presented in Figs. 2 and 3. A
Table 1 Baseline characteristics between groups

\begin{tabular}{|c|c|c|c|}
\hline Variable & $\begin{array}{l}\text { Erlotinib } \\
(n=112)\end{array}$ & $\begin{array}{l}\text { Gefitinib } \\
(n=115)\end{array}$ & $p$-value \\
\hline Age at onset (years) & $\begin{array}{l}58.4 \pm \\
19.52\end{array}$ & $\begin{array}{l}58.5 \pm \\
20.13\end{array}$ & $0.212^{a}$ \\
\hline Sex & & & $0.846^{b}$ \\
\hline Female & 85 & 86 & \\
\hline Male & 27 & 29 & \\
\hline Smoking status & & & $0.644^{c}$ \\
\hline Never a smoker & 67 & 65 & \\
\hline Former smokers & 23 & 26 & \\
\hline Current smokers & 22 & 24 & \\
\hline Largest size of brain metastasis & & & $0.841^{c}$ \\
\hline$\leq 10 \mathrm{~mm}$ & 26 & 28 & \\
\hline$>10 \mathrm{~mm}$ & 86 & 87 & \\
\hline Number of brain metastasis & & & $0.764^{c}$ \\
\hline$\leq 3$ & 65 & 69 & \\
\hline$>3$ & 47 & 46 & \\
\hline ECOG performance status & & & $0.838^{c}$ \\
\hline 0 & 33 & 35 & \\
\hline 1 & 46 & 43 & \\
\hline 2 & 25 & 27 & \\
\hline 3 & 8 & 10 & \\
\hline $\begin{array}{l}\text { Neurological symptoms before } \\
\text { the initiation of TKls }\end{array}$ & & & $0.352^{c}$ \\
\hline Nausea & 6 & 5 & \\
\hline Headache & 3 & 3 & \\
\hline $\begin{array}{l}\text { Depressed level of } \\
\text { consciousness }\end{array}$ & 2 & 2 & \\
\hline Gait disturbance & 1 & 0 & \\
\hline Muscle weakness & 0 & 1 & \\
\hline Dizziness & 1 & 1 & \\
\hline Urinary retention & 1 & 1 & \\
\hline Cognitive disturbance & 2 & 2 & \\
\hline Memory impairment & 1 & 2 & \\
\hline Blurred vision & 2 & 1 & \\
\hline
\end{tabular}

${ }^{\mathrm{a} A n a l y s e d}$ using independent-samples t-test. ${ }^{\mathrm{b}}$ Analysed using chi-squared test. 'Analysed using the Mann-Whitney test. ECOG Eastern Cooperative Oncology Group. TKIs Tyrosine kinase inhibitors

statistically significant difference was detected in median PFS and median OS between groups. Multivariate analysis, after adjusting for age, sex and time span of smoking history, indicated that erlotinib-treated patients had a 36-month PFS rate of 64\% compared with $53 \%$ for gefitinib-treated patients $(\mathrm{HR}=0.28$; $95 \% \mathrm{CI}: 0.17-0.41$; $p=0.013$ ); erlotinib-treated patients had a 36-month OS of $58.3 \%$ compared with $49.1 \%$ for gefitinib-treated patients (HR: $0.21 ; 95 \%$ CI: 0.15 to $0.37 ; p=0.012$ ). 
Table 2 Survival analysis at final follow-up

\begin{tabular}{|c|c|c|c|}
\hline Variable & $\begin{array}{l}\text { Erlotinib } \\
(n=112)\end{array}$ & $\begin{array}{l}\text { Gefitinib } \\
(n=115)\end{array}$ & $p$-value \\
\hline median PFS (months) & $\begin{array}{l}10.8 \text { (range, } \\
0-21.3 \text { ) }\end{array}$ & $\begin{array}{l}8.4 \text { (range, } \\
0-20.5)\end{array}$ & $0.014^{* a}$ \\
\hline median OS (months) & $\begin{array}{l}\text { 28.3(range, } \\
3.6-36.2 \text { ) }\end{array}$ & $\begin{array}{l}\text { 25.0(range, } \\
3.3-36.3)\end{array}$ & $0.033^{* a}$ \\
\hline Deaths, No. & 50 & 67 & $0.04^{* b}$ \\
\hline Age(y) & $68.1 \pm 8.73$ & $67.7 \pm 9.34$ & $0.175^{c}$ \\
\hline Sex & & & $0.133^{b}$ \\
\hline Female & 30 & 49 & \\
\hline Male & 20 & 18 & \\
\hline Smoking status & & & $0.770^{d}$ \\
\hline Never a smoker & 30 & 44 & \\
\hline Former smokers & 13 & 10 & \\
\hline Current smokers & 7 & 13 & \\
\hline $\begin{array}{l}\text { Largest size of brain } \\
\text { metastasis }\end{array}$ & & & $0.326^{d}$ \\
\hline$\leq 10 \mathrm{~mm}$ & 11 & 10 & \\
\hline$>10 \mathrm{~mm}$ & 39 & 57 & \\
\hline Number of brain metastasis & & & $0.467^{d}$ \\
\hline$\leq 3$ & 22 & 25 & \\
\hline$>3$ & 28 & 42 & \\
\hline ECOG performance status & & & $0.177^{d}$ \\
\hline 0 & 6 & 8 & \\
\hline 1 & 15 & 30 & \\
\hline 2 & 21 & 22 & \\
\hline 3 & 8 & 7 & \\
\hline
\end{tabular}

*Statistically significant. ${ }^{a}$ Analysed using the log-rank test; ${ }^{b}$ Analysed using independent-samples t-test; ${ }^{c}$ Analysed using chi-squared test; ${ }^{\mathrm{d}}$ Analysed using the Mann-Whitney test. PFS progression-free disease-free survival; OS overall survival; ECOG Eastern Cooperative Oncology Group

\section{Discussion}

In the current study, Asian patients with positive exon 19 EGFR-mutant lung adenocarcinoma and newly diagnosed brain metastases who initially received peroral administration of $150 \mathrm{mg} / \mathrm{d}$ erlotinib or $250 \mathrm{mg} / \mathrm{d}$ gefitinib were followed for a mean of 36 months, and the most important finding was that erlotinib was associated with a significantly longer OS and more prolonged PFS than gefitinib.

This has increasingly become a consensus that the supreme benefit of EGFR-TKI therapy occurred in patients with EGFR-mutant lung adenocarcinoma and brain metastases [11, 15-19]. The evidence in the previous literature regarding the optimal treatment strategy for the initial management of Asia patients with metastatic EGFR-mutant lung adenocarcinoma was questionable [5, $6,17]$, although there are limited randomized trials directing this therapy. To date, there was no solid evidence that gefitinib or erlotinib had less efficacy than afatinib in first-line treatment of patients with EGFR-mutant lung adenocarcinoma and brain metastases [1-4, 12]. Several studies indicated that gefitinib may be superior to erlotinib, but the finding was based on low event numbers and small sample sizes [20-22]. Our findings were in line with previous prospective trials that the response rates to EGFR-TKI therapy in stage IV lung adenocarcinoma patients harbouring exon 19 EGFR mutation ranged from 60 to $70 \%[13,22]$. Moreover, more studies that compared both OS and PFS between erlotinib and gefitinib in stage IV exon 19 EGFR-mutant lung adenocarcinoma patients after completion of all standard adjuvant chemotherapy and/or radiation therapy also showed similar outcomes [5, 12, 21]. Previous studies established erlotinib was superior to gefitinib in advanced EGFR-mutated patients with leptomeningeal metastases from lung adenocarcinomas that progressed during gefitinib therapy but responded to erlotinib [3, 15, 20, 21].

A retrospective multicenter study by Fan et al. [5] exhibited that median PFS of gefitinib and erlotinib groups was 3.6 and 4.6 months, respectively $(p<0.027)$. Median OS of gefitinib and erlotinib groups was 9.6 and 10.7 months, respectively $(p<0.013)$. Nevertheless, a previous meta-analysis reported by Normando et al. [23] demonstrated no significant difference in the PFS and OS of erlotinib or gefitinib in patients with EGFRmutant lung adenocarcinoma and brain metastases. Recent studies [20,24] exhibited that the PFS and OS of gefitinib-treated patients was significantly lower than that of erlotinib-treated patients. In exploratory analysis of EGFR-mutated patients, gefitinib failed to generate a PFS or OS benefit $[6,25]$. Considering this was an underpowered study that was terminated early with some cases undergoing a short treatment time, the results did not seem to draw conclusions about the impact of erlotinib or gefitinib. Nevertheless, evidencebased medicine analysis [26] exhibited that the PFS and OS of erlotinib-treated young patients (45-55 years old) failed to be superior to gefitinib-treated young patients. Several studies have reported that gefitinib might be a soothing choice for the initial treatment of patients with EGFR-mutant lung adenocarcinoma and newly diagnosed brain metastases [5, 27, 28]. However, another considered problem is that the results after a failed erlotinib or gefitinib are relatively controversial [4]. Currently, there is no consensus about which drug to use in Asian patients with EGFR-mutant lung adenocarcinoma and brain metastases [29]. In China, $80 \%$ of patients prefer receiving gefitinib over erlotinib for brain metastases following EGFR-mutant lung adenocarcinoma. The main reason is that gefitinib has a price advantage, and medical insurance can be reimbursed. Only when gefitinib resistance occur are they willing to accept erlotinib treatment. Thus, further study is 


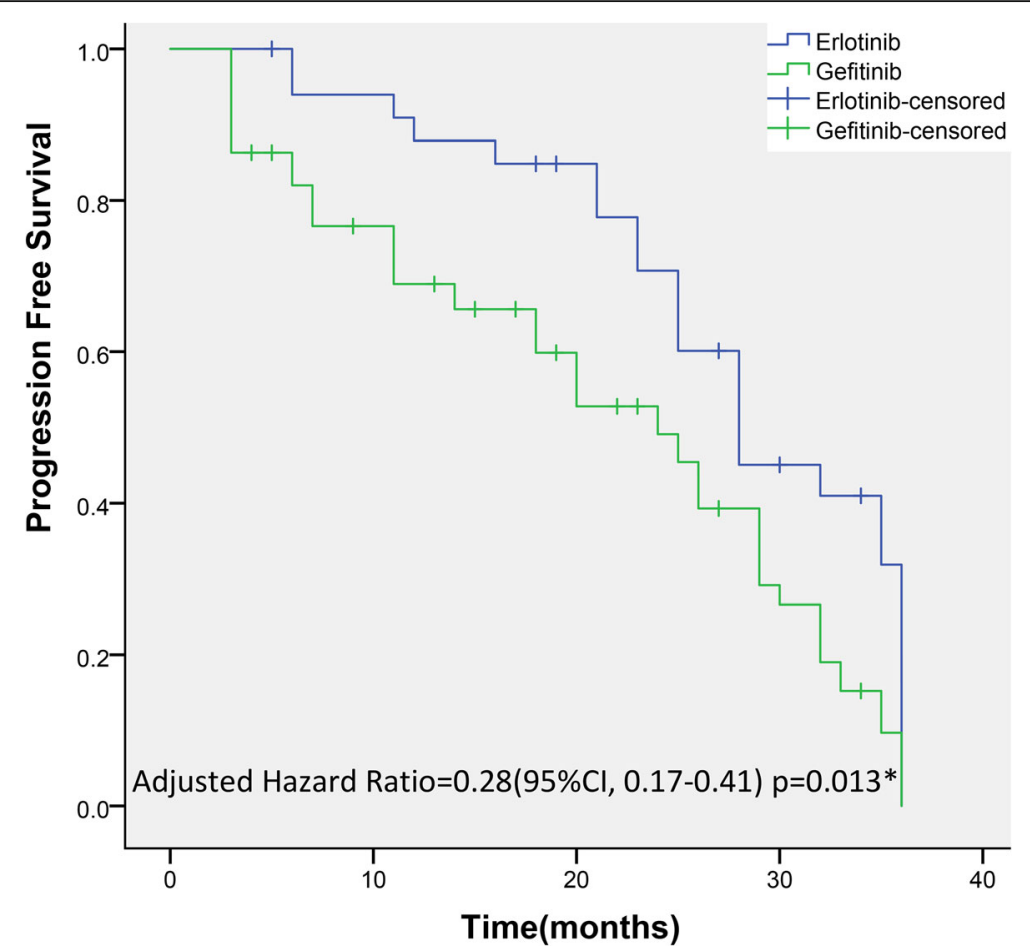

Fig. 2 Kaplan-Meier Curves for PFS. The median PFS was 10.8 months (range, 0-21.3 months) in the erlotinib group and 8.4 months (range, 0-20.5 months) in the gefitinib group. A statistically significant difference was detected in PFS between groups. ${ }^{*}$ The hazard ratio was calculated using the Cox proportional hazards model, with age, sex and time span of smoking history as covariates and gefitinib/erlotinib therapy as the time-dependent factor. With respect to PFS, the results were analysed using the log-rank test $(p=0.014)$

compulsory on the effects of familial exon 19 EGFR mutation on Asian ethnicity. Consequently, whether erlotinib is superior to gefitinib in the treatment of young patients with brain metastases following EGFR-mutant lung adenocarcinoma, a prospective randomized controlled study of larger samples is required for clarification. Noteworthy, any data from EGFR-TKI trials that fail to select patients based on molecular and clinical characteristics and EGFR-mutant presence may be misrepresentative.

Erlotinib, a specific EGFR-TKI, has been shown to improve PFS compared with chemotherapy when given as first-line treatment for Asian patients with NSCLC with activating EGFR mutations [12, 30]. A multicentre, open-label, randomised phase 3 trial (EURTAC) [30] which is the first prospective head-to-head phase 3 study has shown that erlotinib had longer PFS and milder side-effects than standard chemotherapy in non-Asian patients with advanced NSCLC and EGFR mutations. A randomised, phase III study(OPTIMAL, CTONG-0802) [31] comparing erlotinib with chemotherapy as first-line treatment of EGFR mutation-positive advanced NSCLC showed erlotinib should be considered standard first-line treatment of patients with advanced NSCLC and EGFR mutations. Our findings were consistent with the OPTIMAL. In our study, some statistical results could not be obtained when comparing the OS and PFS between groups. One potential explanation may be attributed to that the treatment period of some patients was less than 6 months, related to premature death.

As an EGFR-targeted drug for effective treatment of advanced NSCLC, erlotinib's main drug-related toxicity was rash, mostly mild to moderate [32]. The rashes in most patients in this study were comparable to those in previous studies [11,31], and the symptoms tended to improve after appropriate treatment. The incidence of grade 3 or 4 adverse events was low. No patient reduced or discontinued treatment due to intolerable adverse reactions.

This study should be interpreted considering important limitations. Firstly, the most important limitation is the retrospective nature, which limits the level of evidence. Many cases were excluded from the analysis owing to lack of baseline data. The excluded cases may introduce bias which is scarcely possible to account for and fails to be representative of the larger sample. Secondly, our findings were also limited by the frequency and length of follow-up. Thirdly, although potential confounders were adjusted by us, other unpredictable factors may also be relevant. 


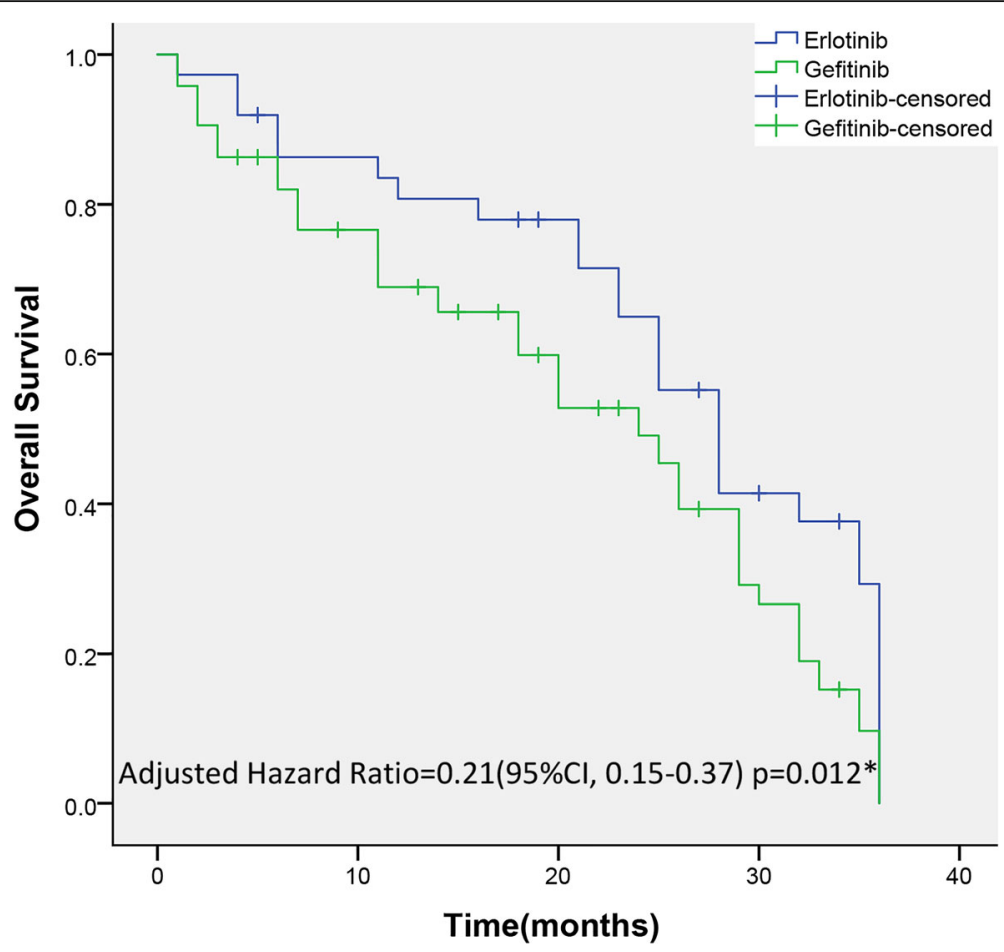

Fig. 3 Kaplan-Meier Curves for OS. The median OS was 28.3 months (range, 3.6-36.2 months) in the erlotinib group and 25.0 months (range, 3.3-36.3 months) in the gefitinib group. There was a statistically significant difference in OS between groups. * The hazard ratio was calculated using the Cox proportional hazards model, with age, sex and time span of smoking history as covariates and gefitinib/erlotinib therapy as the time-dependent factor. With respect to the OS, the results were analysed using the log-rank test $(p=0.033)$

\section{Conclusion}

For Asian patients with EGFR-mutant lung adenocarcinoma and brain metastases, erlotinib was associated with a more prolonged PFS and a significantly longer OS compared with gefitinib. Patients with gefitinib-resistant brain metastases appear to be more suitable for treatment with erlotinib. In addition, if gefitinib or erlotinib were to be assessed again in the adjuvant setting, the proper duration of drug use to maximise efficacy but minimise adverse reaction should not be disregarded. Further follow-up is deserved to verify whether previous findings persist over a longer period.

\section{Abbreviations}

Cl: confidence interval; ECOG: Eastern Cooperative Oncology Group; EGFR: epidermal growth factor receptor; HR: hazard ratio; IQR: interquartile range; NSCLC: non-small cell lung cancer; OS: overall survival; PFS: progression-free survival; RCTs: randomized controlled trials; SD: standard deviation; TKls: tyrosine kinase inhibitors

\section{Acknowledgements}

The authors would like to acknowledge Dr. Huihan Wang for her assistance with the technical help.

\section{Funding}

Funding for this research was received from the Shanghai Municipal Health and Family Planning Commission Fund Project (Grant No. 201640057), and the National Natural Science Foundation of China (Grant No. 81600065; 81270011; 81472125).

\section{Availability of data and materials}

The datasets used and/or analysed during the current study are available from the corresponding author on reasonable request.

\section{Authors' contributions}

YJ: Planning and study design, study execution, writing-initial draft, and writing-final revision. JZ and JH: Statistical analysis/interpretation, writinginitial draft, and writing-final revision. BX: Planning and study design. NL and LC: Study execution. MZ: Study execution. JZ and LC: Data collection and study execution. YJ and MZ: Writing-initial draft, and writing-final revision. Each author contributed important intellectual content during the drafting or revision of the article and accepts accountability for the overall work by ensuring that questions pertaining to the accuracy or integrity of any portion of the work were appropriately investigated and resolved. All authors read and approved the final manuscript.

\section{Ethics approval and consent to participate}

The study was conducted in accordance with the Declaration of Helsinki. The histological samples collected were analysed and the clinical data were retrieved with the endorsement of the Institutional Review Boards and Ethical Review Boards of The Affiliated hospital of Hebei University; The First Affiliated Hospital, Sun Yat-sen University; Jinshan Hospital, Fudan University; The Central Hospital of Wuhan, Tongji Medical College, Huazhong University of Science and Technology. The informed consent from participants was waived by Ethical Review Boards.

\section{Consent for publication}

Not applicable as no identifying personal information is being published in this manuscript.

\section{Competing interests}

The authors declare that they have no competing interests. 


\section{Publisher's Note}

Springer Nature remains neutral with regard to jurisdictional claims in published maps and institutional affiliations.

\section{Author details}

'Department of Neurology, The Affiliated hospital of Hebei University, Yuhua East Road No. 212, Baoding 071000, Hebei, China. ${ }^{2}$ Department of Respiratory Medicine, The First Affiliated Hospital, Sun Yat-sen University, No. 58, Zhongshan 2nd Road, Yuexiu District, Guangzhou 510080, China. ${ }^{3}$ Department of Anesthesiology, The Central Hospital of Wuhan, Tongji Medical College, Huazhong University of Science and Technology, Gusao Road No. 16, Jianghan District, Wuhan 430014, Hubei, China. ${ }^{4}$ Department of Thoracic surgery, The First Affiliated Hospital, Sun Yat-sen University, No. 58, Zhongshan 2nd Road, Yuexiu District, Guangzhou 510080, China. ${ }^{5}$ Department of Orthopaedics, Jinshan Hospital, Fudan University, Longhang Road No. 1508, Jinshan District, Shanghai City 201508, China.

Received: 25 August 2018 Accepted: 31 October 2018

Published online: 20 November 2018

\section{References}

1. Yomo S, Oda K. Impacts of EGFR-mutation status and EGFR-TKI on the efficacy of stereotactic radiosurgery for brain metastases from non-small cell lung adenocarcinoma: a retrospective analysis of 133 consecutive patients. Lung Cancer. 2018;119:120-6.

2. Urata Y, Katakami N, Morita S, Kaji R, Yoshioka H, Seto T, et al. Randomized phase III study comparing Gefitinib with Erlotinib in patients with previously treated advanced lung adenocarcinoma: WJOG 5108L. J Clin Oncol. 2016; 34(27):3248

3. Ostoros G, Sarosi V, Mandoky L, Pinter F, Schwab R, Petak I, et al. Effectiveness of erlotinib in lung adenocarcinomas with classic and alternative EGFR mutations detected by Roche 454 GS-FLX next-generation sequencing (NGS) J Clin Oncol. 2013;31(15).

4. Lee CN, Chen HY, Liu HE. Favorable response to Erlotinib in a lung adenocarcinoma with both epidermal growth factor receptor exon 19 deletion and K-ras G13D mutations. J Clin Oncol. 2010;28(7):E111-E2.

5. Fan WC, Yu CJ, Tsai CM, Huang MS, Lai CL, Hsia TC, et al. Different efficacies of Erlotinib and Gefitinib in Taiwanese patients with advanced non-small cell lung Cancer a retrospective multicenter study. J Thorac Oncol. 2011;6(1):148-55.

6. Janjigian YY, Park BJ, Kris MG, Miller VA, Riely GJ, Zheng J, et al. Impact on disease-free survival of adjuvant erlotinib or gefitinib in patients with resected lung adenocarcinomas that harbor epidermal growth factor receptor (EGFR) mutations. J Clin Oncol. 2009;27(15).

7. Kashima J, Okuma Y, Miwa M, Hosomi Y. Retrospective analysis of survival in patients with leptomeningeal carcinomatosis from lung adenocarcinoma treated with erlotinib and gefitinib. Jpn J Clin Oncol. 2017;47(4):357-62. https://doi.org/10.1093/jjco/hyw206.

8. Gerber NK, Yamada Y, Rimner A, Shi WJ, Riely GJ, Beal K, et al. Erlotinib versus radiation therapy for brain metastases in patients with EGFR-mutant lung adenocarcinoma. Int J Radiat Oncol Biol Phys. 2014;89(2):322-9.

9. Zhuang H, Yuan Z, Wang J, Zhao L, Wang P. Phase II study of WBRT with or without concurrent Erlotinib for patients with brain metastases from lung adenocarcinoma. Int J Radiat Oncol Biol Phys. 2012;84(3):S102.

10. Zhuang HQ, Yuan ZY, Wang J, Zhao L, Pang QS, Wang P. Phase II study of whole brain radiotherapy with or without erlotinib in patients with multiple brain metastases from lung adenocarcinoma. Drug Des Dev Ther. 2013;7:1179-86.

11. Janjigian YY, Park BJ, Zakowski MF, Ladanyi M, Pao W, D'Angelo SP, et al. Impact on disease-free survival of adjuvant Erlotinib or Gefitinib in patients with resected lung adenocarcinomas that harbor EGFR mutations. J Thorac Oncol. 2011;6(3):569-75.

12. Yang CJ, Hung JY, Tsai MJ, Wu KL, Liu TC, Chou SH, et al. The salvage therapy in lung adenocarcinoma initially harbored susceptible EGFR mutation and acquired resistance occurred to the first-line gefitinib and second-line cytotoxic chemotherapy. BMC Pharmacol Toxicol. 2017;18.

13. Bean J, Brennan C, Shih JY, Riely G, Viale A, Wang L, et al. MET amplification occurs with or without T790M mutations in EGFR mutant lung tumors with acquired resistance to gefitinib or erlotinib. Mol Cancer Ther. 2007;6(12):3333S-4S.

14. Asami K, Kawahara M, Atagi S, Kawaguchi T, Okishio K. Duration of prior gefitinib treatment predicts survival potential in patients with lung adenocarcinoma receiving subsequent erlotinib. Lung Cancer. 2011;73(2):211-6.
15. Cardona AF, Arrieta O, Zapata MI, Rojas L, Wills B, Reguart N, et al. Acquired resistance to Erlotinib in EGFR mutation-positive lung adenocarcinoma among Hispanics (CLICaP). Target Oncol. 2017;12(4):513-23.

16. Pan QL, Pao W, Ladanyi M. Rapid polymerase chain reaction-based detection of epidermal growth factor receptor gene mutations in lung adenocarcinomas. J Mol Diagn. 2005;7(3):396-403.

17. Pao W, Wang TY, Riely GJ, Miller VA, Pan QL, Ladanyi M, et al. KRAS mutations and primary resistance of lung adenocarcinomas to gefitinib or erlotinib. PLoS Med. 2005;2(1):57-61.

18. Sholf LM, Janne PA, Jackman DM, Joshi VA. Lindeman NI. EGFR kinase domain mutations, but not copy number, predict response to erlotinib and gefitinib in patients with advanced lung adenocarcinoma. J Mol Diagn. 2007;9(5):692.

19. Tanaka K, Hida T, Oya Y, Oguri T, Yoshida T, Shimizu J, et al. EGFR mutation impact on definitive concurrent Chemoradiation therapy for inoperable stage III adenocarcinoma. J Thorac Oncol. 2015;10(12):1720-5.

20. Yang JJ, Zhou Q, Yan HH, Zhang XC, Chen HJ, Tu HY, et al. A phase III randomised controlled trial of erlotinib vs gefitinib in advanced non-small cell lung cancer with EGFR mutations. Br J Cancer. 2017;116(5):568-74.

21. Tetsumoto S, Osa A, Kijima T, Minami T, Hirata H, Takahashi R, et al. Two cases of leptomeningeal metastases from lung adenocarcinoma which progressed during gefitinib therapy but responded to erlotinib. Int J Clin Oncol. 2012;17(2):155-9.

22. Xu Y, Liu HY, Chen J, Zhou QH. Acquired resistance of lung adenocarcinoma to EGFR-tyrosine kinase inhibitors gefitinib and erlotinib. Cancer Biol Ther. 2010; 9(8):572-82.

23. Normando SRC, Cruz FM, del Giglio A. Cumulative meta-analysis of epidermal growth factor receptor-tyrosine kinase inhibitors as first-line therapy in metastatic non-small-cell lung cancer. Anti-Cancer Drugs. 2015;26(9):995-1003.

24. Shen YC, Tseng GC, Tu CY, Chen WC, Liao WC, Chen WC, et al. Comparing the effects of afatinib with gefitinib or Erlotinib in patients with advancedstage lung adenocarcinoma harboring non-classical epidermal growth factor receptor mutations. Lung Cancer. 2017;110:56-62.

25. Katayama T, Shimizu J, Suda K, Onozato R, Fukui T, Ito S, et al. Efficacy of Erlotinib for brain and leptomeningeal metastases in patients with lung adenocarcinoma who showed initial good response to Gefitinib. J Thorac Oncol. 2009:4(11):1415-9.

26. Wu SG, Shih JY, Yu CJ, Yang PC. Lung adenocarcinoma with good response to erlotinib after gefitinib treatment failure and acquired T790M mutation. J Thorac Oncol. 2008;3(4):451-2.

27. Yu SF, Wang Y, Li JL, Hao XZ, Wang B, Wang ZP, et al. Gefitinib versus erlotinib as salvage treatment for lung adenocarcinoma patients who benefited from the initial gefitinib: a retrospective study. Thorac Cancer. 2013:4(2):109-16.

28. Xing PY, Li JL, Shi YK, Zhang XR. Recurrent response to advanced lung adenocarcinoma with erlotinib developing leptomeningeal metastases during gefitinib therapy and two case reports. Thorac Cancer. 2014;5(1):38-42.

29. Chang $\mathrm{CH}$, Lee $\mathrm{CH}$, Wang JY. Gefitinib or Erlotinib for previously treated lung adenocarcinoma: which is superior? J Clin Oncol. 2017;35(12):1374.

30. Rosell R, Carcereny E, Gervais R, Vergnenegre A, Massuti B, Felip E, et al. Erlotinib versus standard chemotherapy as first-line treatment for European patients with advanced EGFR mutation-positive non-small-cell lung cancer (EURTAC): a multicentre, open-label, randomised phase 3 trial. Lancet Oncol. 2012;13(3):239-46.

31. Zhou C, Wu YL, Chen G, Feng J, Liu XQ, Wang C, et al. Final overall survival results from a randomised, phase III study of erlotinib versus chemotherapy as first-line treatment of EGFR mutation-positive advanced non-small-cell lung cancer (OPTIMAL, CTONG-0802). Ann Oncol. 2015;26(9):1877-83.

32. Fukui T, Otani S, Hataishi R, Jiang SX, Nishii Y, lgawa S, et al. Successful rechallenge with erlotinib in a patient with EGFR-mutant lung adenocarcinoma who developed gefitinib-related interstitial lung disease. Cancer Chemother Pharmacol. 2010;65(4):803-6. 